\title{
High-power ECH and fully non-inductive operation with ECCD in the TCV tokamak
}

S Coda, T P Goodman, M A Henderson, F Hofmann, Z A Pietrzyk, O Sauter, S Alberti, C Angioni, K Appert, R Behn, P Blanchard, P Bosshard, R Chavan, A Degeling, B P Duval, D Fasel, A Favre, I Furno, P Gomez, P Gorgerat, J-P Hogge, P-F Isoz, B Joye, P Lavanchy, J B Lister, X Llobet, J-C Magnin, A Manini, B Marlétaz, P Marmillod, Y Martin, An Martynov, J-M Mayor, J Mlynar, J-M Moret, P Nikkola, P J Paris, A Perez, Y Peysson†, R A Pitts, A Pochelon, H Reimerdes, J H Rommers, E Scavino, A Sushkov $\ddagger$, G Tonetti, M Q Tran, H Weisen and A Zabolotsky

Centre de Recherches en Physique des Plasmas, Association EURATOM-Confédération Suisse, Ecole Polytechnique Fédérale de Lausanne, CH-1015 Lausanne, Switzerland † Département de Recherches sur la Fusion Contrôlée, Association EURATOM-CEA, CEA/Cadarache, 13108 Saint Paul-lez-Durance Cédex, France

$\ddagger$ INF, Russian Research Center, Kurchatov Institute, Moscow, Russian Federation

Received 16 June 2000

\begin{abstract}
Experiments with high-power electron cyclotron heating $(\mathrm{ECH})$ and current drive (ECCD) in the TCV tokamak are discussed. Power up to $2.7 \mathrm{MW}$ from six gyrotrons is delivered to the tokamak at the second-harmonic frequency $(82.7 \mathrm{GHz})$ in $\mathrm{X}$-mode. The power is transmitted to the plasma by six independent launchers, each equipped with steerable mirrors that allow a wide variety of injection angles in both the poloidal and toroidal directions. Fully non-inductive operation of the tokamak has been achieved in steady state, for the full $2 \mathrm{~s}$ gyrotron pulse duration, by co-ECCD with a highest current to date of $210 \mathrm{kA}$ at full power. The experimentally measured ECCD efficiency agrees well with predictions obtained from linear modelling. We have observed that the highest global efficiency attainable at a given power is limited by stability constraints. While the efficiency is maximum on the magnetic axis, a disruptive MHD instability occurs when the width of the deposition profile is lower than a minimum value, which increases with total power. Many ECCD discharges display a high level of electron energy confinement, enhanced by up to a factor of two over the Rebut-Lallia-Watkins (RLW) scaling law, which by contrast is well satisfied in ohmic conditions. The longest confinement times (up to four times RLW) are observed with central counter-ECCD. Central electron heat diffusivities comparable to ohmic levels are obtained in these scenarios, with electron temperatures in excess of $10 \mathrm{keV}$.
\end{abstract}

\section{Introduction}

Electron cyclotron heating $(\mathrm{ECH})$ is an attractive technique for supplying energy to plasmas in thermonuclear regimes, owing to its high efficiency and localized deposition and to the high power throughput and modest space requirements of the launching structures. In recent years, substantial advances in the development of long-pulse, high-power gyrotron sources have significantly broadened the scope of this technique in the context of nuclear fusion research, particularly in tokamaks. ECH experiments can now be performed in quasi-steady-state, i.e. for times longer than all characteristic times in the plasma, and with power levels far exceeding the ohmic heating component [1]. 
Electron cyclotron waves are also utilized for current drive (ECCD). This method is ideally suited for current profile control, thanks to its good efficiency combined with the high degree of localization and ease of positioning of the current source. Profile control is a key ingredient in prospective reactor operation, both in the conventional tokamak configuration, for MHD stabilization purposes, and in the so-called 'advanced tokamak' configuration, for optimization of performance in steady state.

The first demonstration of steady-state operation in a tokamak with the toroidal current sustained entirely by ECCD has been recently performed in TCV (tokamak à configuration variable), with an injected power of $1.35 \mathrm{MW}$ and a driven current of $123 \mathrm{kA}$ [2]. In this paper we report on the extension of these studies to power levels of $2.7 \mathrm{MW}$ and driven currents of $210 \mathrm{kA}$.

Enhanced central confinement has been observed in TCV with counter-ECCD (antiparallel to the plasma current) on-axis, with central temperatures and confinement times larger by a factor of two than with co-ECCD or pure ECH. With the aid of numerical transport modelling, this effect has been attributed to the inhibition of sawtooth oscillations achieved with this configuration, combined with heating in the centre where diffusivity is lowest [3]. More recently, different combinations of launching geometries, ECCD components and power waveforms have been devised to retain the high confinement while improving the MHD stability properties of the plasma [4]. The common factor in all these scenarios is the presence of a counter-ECCD component on-axis.

This paper is organized as follows. Section 2 contains a brief description of the TCV tokamak and of the ECH/ECCD system, accompanied by an overview of recent results. Studies of fully non-inductive discharges with co-ECCD are discussed in section 3. In section 4 an account of our confinement studies is given, followed by concluding remarks in section 5 .

\section{Experimental set-up and overview of ECH studies}

The TCV tokamak is a medium-sized device, with major radius $R=0.88 \mathrm{~m}$, minor radius $a=0.25 \mathrm{~m}$ and a maximum vacuum magnetic field $B_{\phi}=1.43 \mathrm{~T}$; plasma currents up to $1 \mathrm{MA}$ have been achieved. The highly elongated vacuum vessel is a distinguishing feature of TCV and is designed to accommodate a wide variety of plasma shapes [5]: edge elongations up to 2.70 and triangularities between -0.7 and +0.9 have already been achieved in limiter plasmas.

The second-harmonic, X-mode (X2) ECH system comprises six gyrotrons with a 2 s pulse capability, each connected to a matching optics unit (MOU) for coupling into an evacuated waveguide; the MOU also houses a universal polarizer, which allows the selection of the appropriate X-mode polarization for each launching configuration. The power rating of each gyrotron-MOU unit is $0.47 \mathrm{MW}$. At the end of each waveguide is a launcher with two rotational degrees of freedom to provide maximum flexibility in the aiming of the injected beam. The poloidal angle can be varied in the course of a plasma discharge, while the toroidal angle can be adjusted between discharges. The average power delivered to the plasma by each launcher is $0.45 \mathrm{MW}$ at a frequency of $82.7 \mathrm{GHz}$ [6].

Ultimately, three additional gyrotrons at the third-harmonic (X3) frequency $(118 \mathrm{GHz})$ will supplement the X2 system, for a total ECH power delivered to the plasma in excess of $4 \mathrm{MW}$. The X3 waves will be launched vertically from the vessel ceiling, in a direction nearly tangential to the resonant surface in order to maximize the single-pass absorption.

The studies discussed in this paper relied crucially on some key diagnostic systems. The line-integrated density is measured by a multichannel far-infrared interferometer. Density and temperature profiles are provided by a Thomson scattering system along a vertical chord with a spacing of $4 \mathrm{~cm}$. A soft $\mathrm{x}$-ray diagnostic composed of nine cameras, each with 20 detectors, 
provides complete coverage of the poloidal cross section; these measurements are used in conjunction with Thomson scattering data to calculate the average effective ion charge $Z_{\text {eff }}$.

$\mathrm{ECH}$ experiments have been carried out in TCV at progressively increasing powers during the past four years [7]. An accurate knowledge of the aiming direction and of the beam polarization is a prerequisite to studies that rely on the precise localization of the power deposition location. The behaviour of the sawtooth oscillations in the plasma has been found to depend sensitively on the heating location relative to the position of the flux surface on which the safety factor $q$ is equal to unity, as well as on the local power density and current drive component. This dependence is highly reproducible. A sweep of the poloidal injection angle of each launcher thus yields a very accurate measurement of the difference in angle between launchers, reflected in a precision of $\pm 3 \mathrm{~mm}$ in the knowledge of the relative heating location for cases in which refraction effects can be neglected. The precision on the absolute value of the angle is set by the accuracy of the magnetic equilibrium reconstruction, particularly with respect to the position of the $q=1$ surface. In addition, the energy content of the plasma when heating is applied inside the $q=1$ surface depends strongly on the beam polarization, being maximum in pure X-mode: this feature permits a verification of the correctness of the polarization [6].

Amongst the experiments performed with $\mathrm{ECH}$ during the past year, the exploration of $\mathrm{H}$-mode accessibility at the low densities $\left(<4.3 \times 10^{19} \mathrm{~m}^{-3}\right)$ required for X2 propagation deserves special mention. The low-density bound for the $\mathrm{L}-\mathrm{H}$ transition was lowered with the addition of ECH power, from the previous limit of $3.5 \times 10^{19} \mathrm{~m}^{-3}$ to $2.5 \times 10^{19} \mathrm{~m}^{-3}$ in line-averaged density. Remarkably, an inverse (decreasing) dependence of the $\mathrm{L}-\mathrm{H}$ threshold power on the plasma density was documented in these discharges [8].

The MHD stability properties of the plasmas in the presence of ECH have been investigated. The influence of shaping on sawteeth, previously observed in ohmic plasmas, was significantly enhanced by central ECH: the destabilization (shortening) of sawteeth at high elongation and at low or negative triangularity is amplified by the additional heating inside the $q=1$ surface. Numerical analysis has shown these shapes to be unstable to the ideal internal kink. This is consistent with the lower pressure gradients measured inside the $q=1$ surface in these configurations [9]. Also, neoclassical tearing modes were identified in discharges with central co-ECCD and high pressure gradients. It was also shown that a broadening of the power deposition profile inhibits the appearance of the modes, which can degrade the global confinement time by up to $25 \%$ [10].

A particle transport phenomenon, commonly known as 'density pump-out', whereby particles are driven outward by intense central ECH, is observed and has been linked to MHD mode activity at the $q=1$ surface. Since mode stability is itself strongly dependent on shape, pump-out does not occur at high triangularity when the MHD mode activity is suppressed [11].

\section{Fully non-inductive operation with ECCD}

The plasma current in TCV has been driven entirely by co-ECCD, in steady state, with injected powers ranging from 1.35 to $2.7 \mathrm{MW}$. The highest driven current to date is $210 \mathrm{kA}$ at $2.7 \mathrm{MW}$. As shown in figure 1, the current is sustained for the entire $2 \mathrm{~s}$ gyrotron pulse duration, while plasma conditions stabilize over a characteristic time scale of less than $500 \mathrm{~ms}$. This is also the maximum current profile relaxation time, as inferred from the rate of change of the internal inductance after the application of ECCD: the inductance increases, indicating a peaking of the profile. In the single-null diverted discharge shown in figure 1, the line-averaged density is $1.3 \times 10^{19} \mathrm{~m}^{-3}$ and the central temperature during ECCD is approximately $5 \mathrm{keV}$. The bootstrap current fraction, calculated by means of the finite collisionality model of Sauter et al [12], is $8 \%$ in this discharge. 


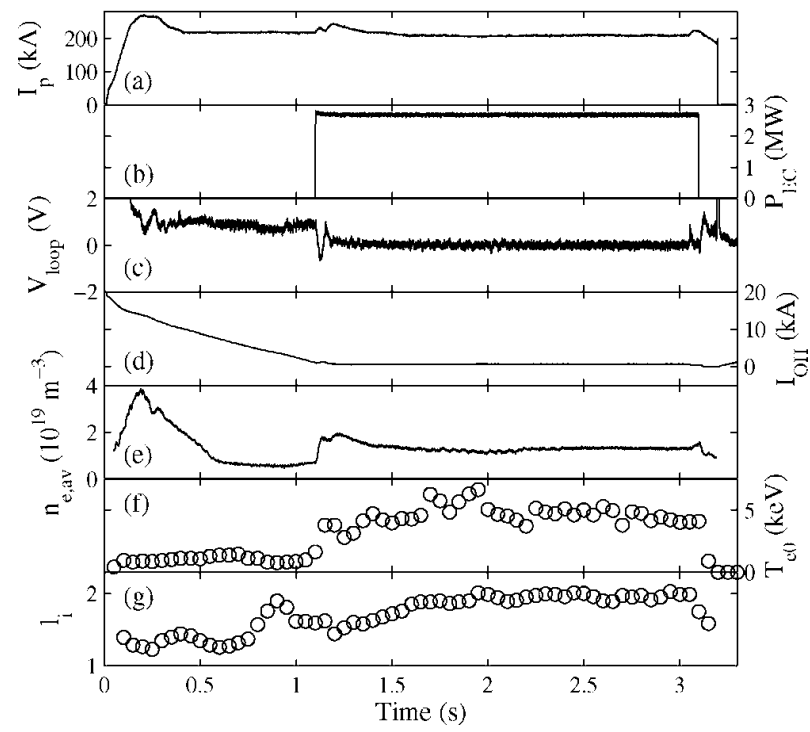

Figure 1. Steady-state, fully non-inductive $210 \mathrm{kA}$ discharge with $2.7 \mathrm{MW}$ of distributed coECCD; the plasma is single-null diverted and has elongation $\kappa_{\text {edge }}=1.6$, triangularity $\delta_{\text {edge }}=0.4$ and safety factor $q_{95}=4.0$ : (a) plasma current, (b) injected electron cyclotron power, (c) loop voltage at plasma boundary, (d) current in the ohmic transformer primary, (e) line-averaged density, (f) peak temperature, $(\mathrm{g})$ internal inductance.

The non-inductive condition is evidenced both by the edge loop voltage averaging zero and, in steady-state conditions, by the current in the primary circuit of the ohmic transformer remaining constant. The latter is in fact the more sensitive and less noisy of the two measurements. In normal tokamak operation, the current in the ohmic coil is controlled by feedback to keep the plasma current equal to a predetermined value. In the present discharges, $50 \mathrm{~ms}$ after the start of the ECCD pulse the feedback configuration is switched so that the ohmic coil current itself is maintained at a preset value while the plasma current is allowed to float; $50 \mathrm{~ms}$ before the end of the pulse, the regular feedback scheme is reinstated. This method guarantees that the inductively driven current component in the plasma is strictly zero, once steady-state conditions are reached and the current in all the shaping coils is also constant.

In a preliminary study, it was found that the current drive efficiency at constant deposition radius increased with increasing toroidal injection angle [13]. Accordingly, in our noninductive discharges the angle was chosen to be the largest compatible with full absorption, minus a safety margin of a few degrees to allow for possible variations in density and thus in beam refraction. The average angle of the six beams to the perpendicular direction is approximately $32^{\circ}$ at the resonance location for the case shown in figure 1 .

The linear ray tracing code TORAY [14], with the Cohen package for current drive estimation [15], has been our main modelling and predictive tool. The theoretical current drive efficiency is linearly dependent on the ratio of the electron temperature to the density, which is generally highest on-axis, and is also strongly dependent on the fraction of particles that are trapped in the magnetic mirror created by the spatial inhomogeneity of the toroidal magnetic field [16]. As the distance from the magnetic axis increases, the trapped particle population increases and the efficiency decreases; in addition, this effect is stronger on the 

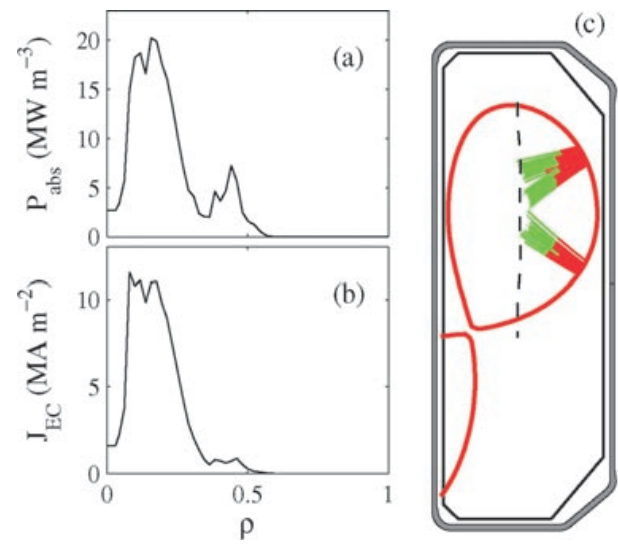

(d)
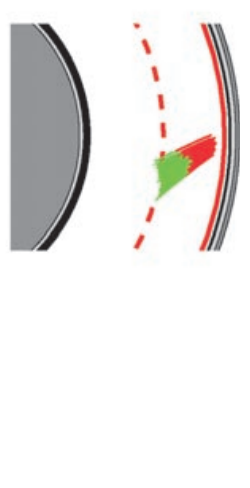

Figure 2. Flux-surface-averaged (a) absorbed power and (b) driven-current density as functions of a normalized radial coordinate proportional to the square root of the plasma volume, for the discharge shown in figure 1 at time $=2.4 \mathrm{~s}$; (c) poloidal and (d) toroidal cross sections of TCV with launching geometry. All data are from TORAY. In (c) and (d) the dashed vertical line is the nominal resonance surface; the lighter ray segments denote the region of $99 \%$ absorption. The average angle between the EC rays and the magnetic field at the resonance is $32^{\circ}$.

low-field side than on the high-field side of the axis. Thus, the efficiency is predicted to be a decreasing function of the minor radius through a combination of these effects.

We have attempted to maximize the efficiency by placing the deposition location of the beam as close to the magnetic axis as possible. However, we found experimentally that an MHD stability limit sets a minimum deposition width below which a disruption occurs before steady state can be attained. The ray trajectories for the case of figure 1 , as well as the radial profiles of the absorbed power density and driven current density, are shown in figure 2 . The width of the deposition profile relative to the plasma centre is the lowest stable width identified over a series of discharges with different launching configurations. It should be noted that the current profile calculated by TORAY for $\rho<0.1$ is extremely sensitive to the details of the equilibrium reconstruction; the hollowness of the profile in figure 2(b), in particular, is not significant within the experimental uncertainties.

The minimum stable deposition profile width is found to increase with increasing injected power. This is consistent with both the pressure and current gradients, which can drive MHD instabilities, increasing with power at constant profile shape. As a result, the maximum global ECCD efficiency achievable appears to be a decreasing function of applied power. This is evidenced by figure 3 , which shows a comparison between the narrowest stable deposition profiles for the $2.7 \mathrm{MW}$ case shown earlier and a case with only $1.35 \mathrm{MW}$. The latter was also a steady-state, fully non-inductive discharge, with a current of $160 \mathrm{kA}$.

The cause of the disruptive instability is not known at present. Low-frequency $(<10 \mathrm{kHz})$ MHD modes are often detected by magnetic probes at the plasma boundary while the plasma conditions relax towards steady state. However, these modes generally disappear when the relaxation is complete; furthermore, no significant degradation in confinement is observed while the mode is present. When the disruption occurs, it is without any immediate precursors and over a time scale of the order of 50-200 $\mu$ s, indicative of an ideal MHD instability. A comparison between a stable and an unstable case at $2.7 \mathrm{MW}$ is shown in figure 4 . The stable case reproduces the one shown in figure 1. The MHD mode can be seen in both discharges. 

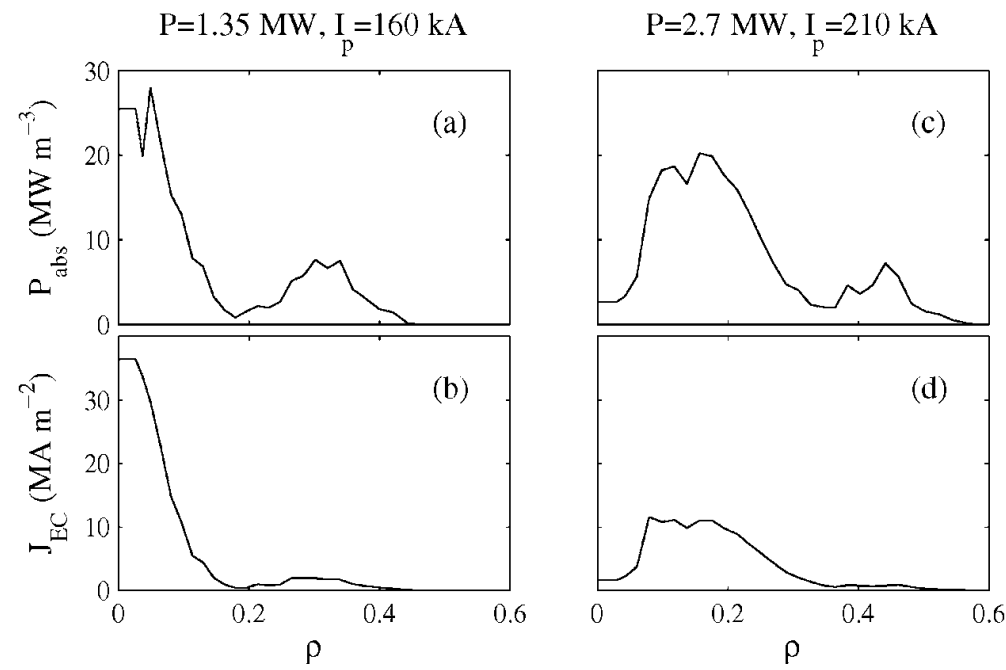

Figure 3. (a) Absorbed power and (b) driven-current density for non-inductive discharge at $160 \mathrm{kA}$ and $1.35 \mathrm{MW}$ power (line-averaged density $n_{\mathrm{e}, \mathrm{av}}=1 \times 10^{19} \mathrm{~m}^{-3}$, central temperature $T_{\mathrm{e} 0}=3.1 \mathrm{keV}, q_{95}=4.8, \kappa_{\text {edge }}=1.5, \delta_{\text {edge }}=0.35$ ); (c) absorbed power and (d) driven-current density for non-inductive discharge at $210 \mathrm{kA}$ and $2.7 \mathrm{MW}$ power $\left(n_{\mathrm{e}, \mathrm{av}}=1.3 \times 10^{19} \mathrm{~m}^{-3}\right.$, $\left.T_{\mathrm{e} 0}=4.7 \mathrm{keV}, q_{95}=4.0, \kappa_{\text {edge }}=1.6, \delta_{\text {edge }}=0.4\right)$. All the profiles are calculated by TORAY.

A paramount issue for ECCD applications is its efficiency, particularly its dependence on the deposition location through trapped particle effects. The normalized efficiency $\eta_{\mathrm{T}}=I_{\mathrm{EC}} R n /\left(P T_{\mathrm{e}}\right)$, where $I_{\mathrm{EC}}$ is the EC-driven current in A, $R$ is the major radius in $\mathrm{m}$, $n$ is the plasma density in $10^{20} \mathrm{~m}^{-3}, P$ is the ECCD power in $\mathrm{W}$ and $T_{\mathrm{e}}$ is the electron temperature in $\mathrm{keV}$, is an important figure of merit, which is theoretically expected to depend only on the trapped particle population and on the effective ion charge $Z_{\text {eff }}$, which in this study is typically between 5 and 6 with no noticeable dependence on power (above $1.35 \mathrm{MW}$ ) or plasma conditions. The EC-driven current in the steady-state non-inductive mode is simply the plasma current minus the calculated bootstrap fraction, which is generally in the order of $10 \%$. Although $\eta_{\mathrm{T}}$ is a local parameter, we have evaluated a global average $\left\langle\eta_{\mathrm{T}}\right\rangle=I_{\mathrm{EC}}\left[\int \mathrm{d} V P_{\mathrm{abs}} T_{\mathrm{e}} /(R n)\right]^{-1}$, where $P_{\mathrm{abs}}$ is the flux-surface-averaged EC power density calculated by TORAY. While only approximate, this procedure is in fact sufficiently accurate for our immediate purposes: owing to the observed flatness of the density profile, the variation in the quantity $T_{\mathrm{e}} /(R n)$ is mostly due to temperature variations and is typically less than $30 \%$ over the region in which $95 \%$ of the power is absorbed. By contrast, by varying the deposition profile, a variation of $\left\langle\eta_{\mathrm{T}}\right\rangle$ by up to a factor of $\sim 3.5$ (between $1.7 \times 10^{-3}$ and $6.0 \times 10^{-3}$ ) has been recorded, as shown in figure 5 . Here, the deposition is generally slightly on the low-field side of the magnetic axis and vertically displaced from the midplane. These preliminary results already constitute clear evidence of the dependence on the minor radius expected from trapped particle effects. In addition, we find excellent quantitative agreement, to within $20 \%$, with the efficiencies predicted by TORAY. It should be noted that in this comparison the spatial averaging plays no role, as the ratio between the experimental and theoretical values is simply the ratio between the respective values of the total driven current. Thus, nonlinear effects do not appear to be significant in the present conditions. This is in conflict with theoretical predictions [17] indicating that an increase in efficiency should be expected in our regime owing 
(a)

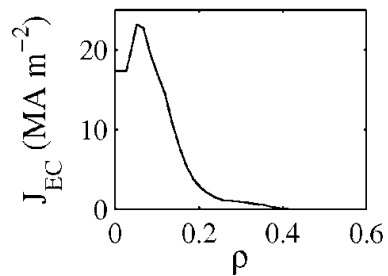

(b)

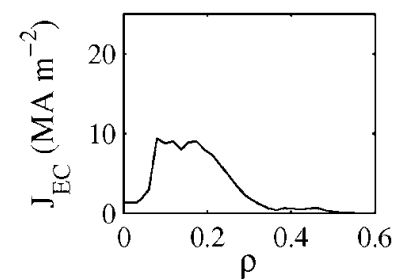

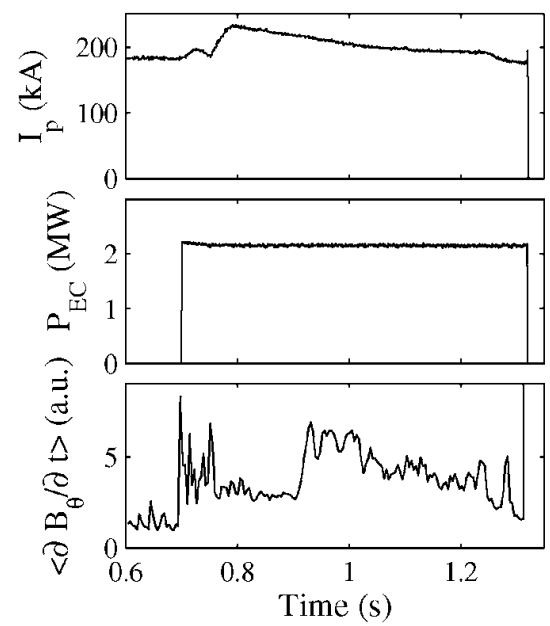
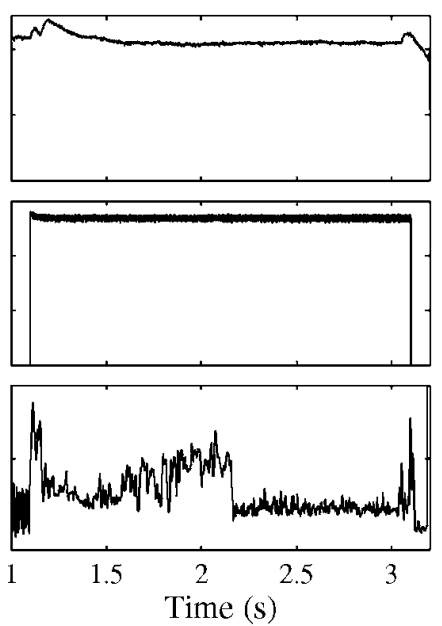

Figure 4. Comparison between (a) an unstable and (b) a stable discharge, both non-inductively driven. At the top are the driven-current density profiles as given by TORAY. The time traces show, from top to bottom, the plasma current, the injected EC power and the rms value of the poloidal magnetic field fluctuations at the inner edge in the range $0-15 \mathrm{kHz}$.

to quasilinear modification of the electron distribution function. A detailed investigation of the local efficiency is planned on TCV in order to address this discrepancy.

\section{Plasma confinement properties in the presence of ECH and ECCD}

Degradation of the plasma energy confinement with the application of additional heating (L-mode) is a well established phenomenon in tokamaks. In hot-electron scenarios, i.e. when heating of the electrons dominates over ion heating, the energy confinement time $\tau_{\mathrm{E}}$ is generally well reproduced by the Rebut-Lallia-Watkins (RLW) scaling law [18]. In TCV, the RLW scaling appears to constitute an especially appropriate benchmark since it is in excellent agreement with experimental measurements in ohmic plasmas and in many instances of Lmode plasmas with ECH [7]. However, in the ECCD experiments described in the previous section, as well as in numerous discharges with pure $\mathrm{ECH}$, we have measured confinement times higher than the RLW scaling by a factor of two or more.

In addition, by applying counter-ECCD on-axis, the confinement time is increased further with respect to cases with co-ECCD or pure ECH by up to a factor of two. Steady-state central temperatures in excess of $10 \mathrm{keV}$ have been measured, and the core electron energy diffusivity is close to or lower than ohmic levels [3]. By driving a negative current on axis, the local current density is decreased: this effect is in competition with the tendency of the 


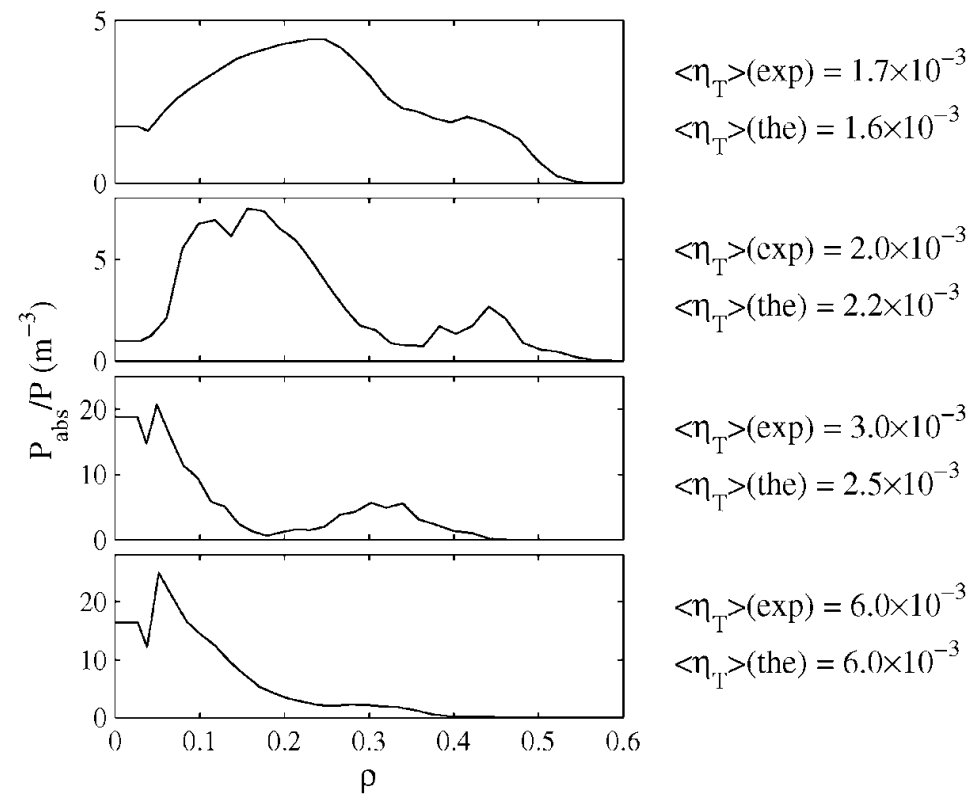

Figure 5. Profiles of normalized power density calculated by TORAY for four different discharges; from top to bottom the deposition becomes increasingly central. Experimental and theoretical (from TORAY) values of the normalized current drive efficiency $\left\langle\eta_{\mathrm{T}}\right\rangle$ are given in units $10^{20} \mathrm{~m}^{-3} \mathrm{~A} \mathrm{~m} \mathrm{~W}^{-1} \mathrm{keV}^{-1}$

current to increase owing to the rising conductivity of the heated plasma core. The first effect dominates for sufficiently large parallel wavenumbers and the safety factor remains above unity. Sawtooth oscillations, which are present in co-ECCD and ECH discharges, therefore do not develop in this case. The transport and equilibrium code PRETOR [19] is capable of reproducing the increase in confinement, indicating that the absence of sawtooth activity may be sufficient to explain this effect. This conclusion is further corroborated by the observation that if sawtooth activity is artificially suppressed in PRETOR in the co-ECCD and ECH cases, the calculated diffusivity decreases dramatically and becomes comparable to that of the counterECCD discharges. It is interesting to note that PRETOR itself is based on the RLW local prescription for the diffusion coefficients; however, the global scaling obtained differs from the RLW global scaling, since the calculated profiles depart from the canonical ones on which the latter is based. An example of this phenomenon is shown in figure 6. Here, an ECCD power ramp from 2 to $2.3 \mathrm{MW}$ was effected; the confinement enhancement factor $\mathrm{H}_{\mathrm{RLW}}=\tau_{\mathrm{E}} / \tau_{\mathrm{RLW}}$ is seen to increase with increasing power. This type of discharge is not always stable and in some cases the confinement is suddenly degraded at a later time or a disruptive instability occurs [4], as in the case shown in figure 6. The core temperature in the high-confinement phase exceeds $10 \mathrm{keV}$, as evidenced further by figure 7 .

Similar or slightly higher confinement enhancement factors have been obtained through a specific dynamical sequence of heating scenarios [4]. First, co-ECCD or ECH is applied off-axis; since the total current is kept constant, the current driven off-axis (by direct drive with ECCD or by conductivity enhancement with ECH) forces the central current to decrease, engendering a similar effect to the on-axis counter-ECCD case and thus keeping the $q<1$ region very small or inhibiting sawteeth altogether. The core diffusivity is reduced in this 


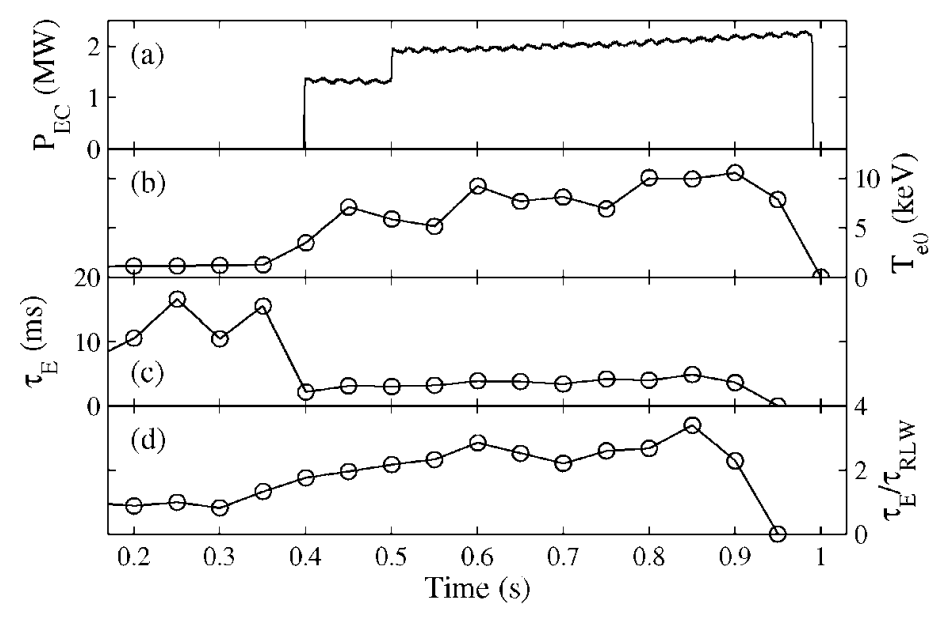

Figure 6. Discharge with counter-ECCD applied on the magnetic axis, with plasma current $I_{\mathrm{p}}=200 \mathrm{kA}, n_{\mathrm{e}, \mathrm{av}}=1-1.3 \times 10^{19} \mathrm{~m}^{-3}, q_{95}=9, \kappa_{\mathrm{edge}}=1.8, \delta_{\text {edge }}=0.3$ : (a) injected EC power, (b) peak temperature, (c) energy confinement time, (d) energy confinement time normalized to the RLW scaling.

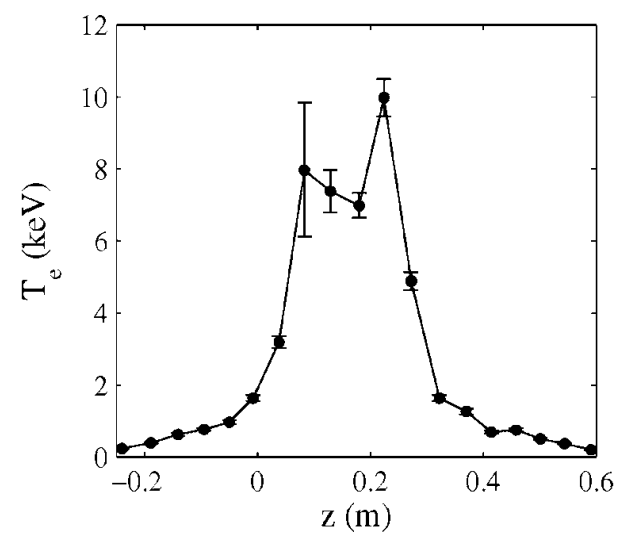

Figure 7. Electron temperature profile measured by Thomson scattering along a vertical chord, for the discharge shown in figure 6 , at time $=0.85 \mathrm{~s}$.

phase. On this baseline of good core confinement, central counter-ECCD is further added after a delay of approximately $300 \mathrm{~ms}$. This sequence is shown in figure 8 with the high $\mathrm{H}_{\mathrm{RLW}}$ factor of 4 attained in the second phase. A case with the two heating schemes applied at the same time is shown here for comparison. The confinement is initially high in this case also, but is later degraded before a steady state can be reached. This suggests that the temporal dynamics of the discharge evolution plays a crucial role in sustaining the enhanced confinement state in a stable manner. A comparison discharge was run with the same time sequence but with the onaxis beams switched to pure ECH mode, and the confinement time was found to be significantly lower, as shown in figure 8 . Note that in all cases $H_{\mathrm{RLW}}=1$ in the initial ohmic phase.

This time-delayed scenario could not be reproduced successfully by PRETOR; in particular, the temperature profiles predicted by the numerical simulation were narrower, 

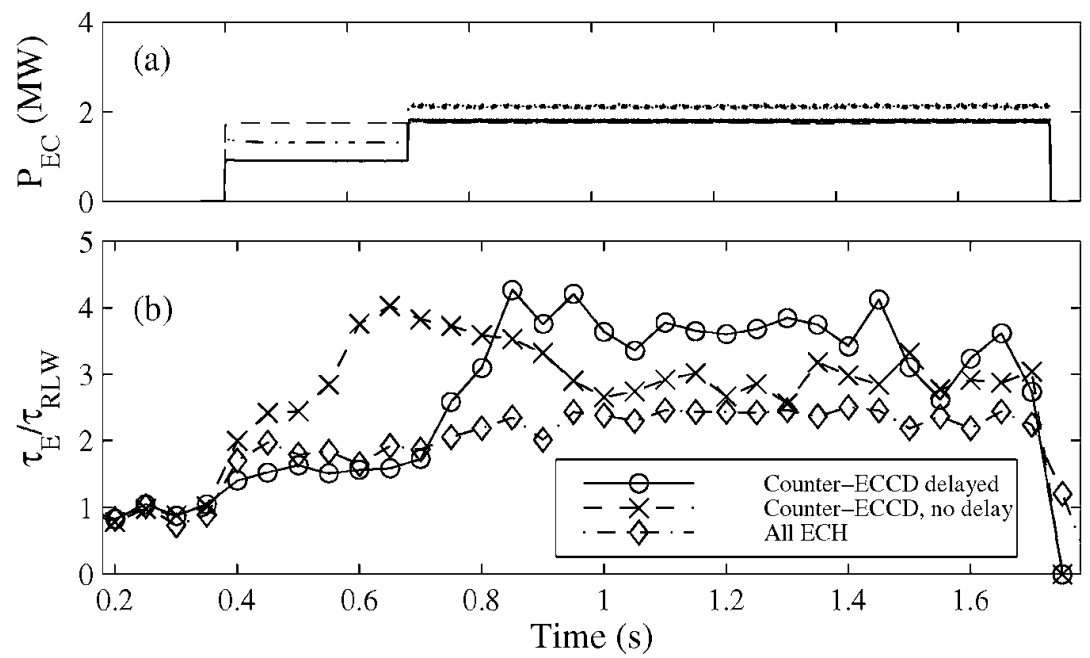

Figure 8. Comparisons of (a) injected EC power and (b) energy confinement time normalized to the RLW scaling, in three different discharges. Plasma parameters are $I_{\mathrm{p}}=200 \mathrm{kA}, n_{\mathrm{e}, \text { av }}=1-$ $1.2 \times 10^{19} \mathrm{~m}^{-3}, q_{95}=6-8, \kappa_{\text {edge }}=1.65, \delta_{\text {edge }}=0.25$. Circles and solid line: off-axis ECH from $0.4 \mathrm{~s}$ followed by on-axis counter-ECCD at $0.7 \mathrm{~s}$; crosses and dashed line: off-axis ECH and on-axis counter-ECCD from $0.4 \mathrm{~s}$; diamonds and dash-dot line: off-axis ECH from $0.4 \mathrm{~s}$ followed by on-axis $\mathrm{ECH}$ at $0.7 \mathrm{~s}$.

while the peak temperature was similar. It should be noted that while the technique described generates a confinement similar to that of the purely on-axis counter-ECCD scenario, the stability properties of the former are better, presumably owing to the broader overall power deposition profile. On-axis counter current drive appears to be an essential ingredient in all the enhanced confinement regimes observed in this study.

\section{Conclusions}

Experiments with steady-state, fully non-inductive ECCD have progressed in TCV from an initial driven current of $123 \mathrm{kA}$ with $1.35 \mathrm{MW}$ to a present highest value of $210 \mathrm{kA}$ with 2.7 MW. The global ECCD efficiency is found to agree well with theoretical predictions from linear modelling, including trapped particle effects, over a broad range of values: in particular, a predicted threefold decrease of the normalized efficiency with increasing deposition width was experimentally verified. MHD stability requirements are empirically found to set a lower limit on the width of the deposition profile and thus an upper limit on the normalized efficiency; the latter decreases with increasing power.

Enhanced electron energy confinement, with central diffusivity of the order of its ohmic counterpart, is obtained with counter-ECCD on-axis, owing to prevention of sawteeth. However, the confinement already exceeds the reference RLW scaling by a factor of two in many sawtoothing discharges with ECH and co-ECCD, primarily as a result of peaked deposition profiles. Improved confinement is also achieved in a more MHD-quiescent configuration by a time-delayed combination of off-axis ECH or co-ECCD and on-axis counter-ECCD. 


\section{Acknowledgment}

This work was supported in part by the Swiss National Science Foundation.

\section{References}

[1] Lloyd B 1998 Plasma Phys. Control. Fusion 40 A119

[2] Sauter O et al 2000 Phys. Rev. Lett. 843322

[3] Pietrzyk Z A et al 2000 Phys. Plasmas 72909

[4] Pietrzyk Z A et al 2000 Proc. 27th EPS Conf. on Controlled Fusion and Plasma Physics (Budapest, 2000) to be published

[5] Hofmann F et al 1994 Plasma Phys. Control. Fusion 36 B277

[6] Henderson M A 1999 CRPP-EPFL Report LRP 643/99

Henderson M A 2000 Proc. 4th Int. Workshop on Strong Microwaves in Plasmas (Nizhny Novgorod, 1999) to be published

[7] Pochelon A et al 1999 Nucl. Fusion 391807

[8] Martin Y et al 1999 Proc. 26th EPS Conf. on Controlled Fusion and Plasma Physics (Maastricht, 1999) Europhys. Conf. Abstr. 23J 1105

[9] Reimerdes H et al 2000 Plasma Phys. Control. Fusion 42629

[10] Reimerdes H et al 2000 Proc. 27th EPS Conf. on Controlled Fusion and Plasma Physics (Budapest, 2000) to be published

[11] Furno I and Weisen H 2000 Proc. 27th EPS Conf. on Controlled Fusion and Plasma Physics (Budapest, 2000) to be published

[12] Sauter O et al 1999 Phys. Plasmas 62834

[13] Sauter O et al 1999 CRPP-EPFL Report LRP 646/99

Sauter $\mathrm{O}$ et al 2000 Fusion engineering and design (Proc. IAEA Technical Committee Meeting on ECRH Physics and Technology for Fusion Devices and EC-11 (Oh-arai, 1999)) to be published

[14] Kritz A H et al 1982 Proc. 3rd Varenna-Grenoble Int. Symp. on Heating in Toroidal Plasmas (Grenoble 1982) vol 2 (Brussels: CEC) p 707

[15] Cohen R H 1987 Phys. Fluids 302442

[16] Alikaev V V and Parail V V 1991 Plasma Phys. Control. Fusion 331639

[17] Harvey R W, McCoy M G and Kerbel GD 1989 Phys. Rev. Lett. 62426

[18] Rebut P H, Lallia P P and Watkins M L 1989 Proc. 12th Int. Conf. on Plasma Physics and Controlled Nuclear Fusion Research (Nice, 1988) vol 2 (Vienna: IAEA) p 191

[19] Boucher D and Rebut P H 1993 Proc. IAEA Tech. Conf. on Advances in Simulation and Modeling in Thermonuclear Plasmas (Montreal 1992) (Vienna: IAEA) p 142

Angioni C, Boucher D, Moret J-M and Sauter O 1998 Theory of Fusion Plasmas (Bologna: Editrice Compositori) p 493 